\title{
Adsorción de plomo $(\mathrm{Pb})$ de aguas contaminadas mediante cáscara de plátano (Musa paradisiaca)
}

\section{Adsorption of lead $(\mathrm{Pb})$ from contaminated aqueous mediums using banana (Musa paradisiaca) peel}

\author{
Ricardo Vejarano, Dr. ${ }^{1, *}$; Julio Gurreonero-Fernández, Ing. ${ }^{1}$; Alberto Castillo-Herrera, Ing. ${ }^{1}$ \\ ${ }^{1}$ Facultad de Ingeniería, Universidad Privada del Norte (UPN), Trujillo, Perú. ricardo.vejarano@upn.edu.pe; \\ julio.gurreonero@upn.edu.pe; acastilloher@hotmail.com;
}

\begin{abstract}
Resumen-El objetivo del estudio fue evaluar la viabilidad para remover plomo $(\mathrm{Pb})$ de aguas contaminadas utilizando biomasa de cáscara de plátano. Se evaluaron el tamaño de partícula de la biomasa y el tiempo de contacto, obteniendo la mayor remoción mediante el tamiz de malla \#60 a los 30 minutos de contacto, con un nivel de remoción de Pb mayor al 98\%. Los resultados sugieren el potencial de remoción de $\mathrm{Pb}$ utilizando cáscara de plátano, el cual con un mínimo tratamiento podría usarse como adsorbente en el tratamiento de aguas contaminadas.
\end{abstract}

Palabras clave: Adsorción de plomo, aguas contaminadas, cáscara de plátano, adsorción de metales pesados.

\begin{abstract}
The aim of the study was to evaluate the feasibility to remove lead (Pb) from contaminated waters using banana peel biomass. Particle size of the biomass and contact time were evaluated, obtaining the higher removal capacity by the \#60 mesh sieve at 30 minutes of contact time, with a removal level of $P b$ greater than $98 \%$. The obtained results suggest the potential for $P b$ removal using banana peel, which with a minimum treatment could be used as adsorbent in the treatment of contaminated waters.
\end{abstract}

Keywords: Lead adsorption, contaminated waters, banana peel, heavy metals adsorption.

\section{INTRODUCCIÓN}

El crecimiento industrial y el deficiente control ambiental en el Perú han generado la contaminación de diversas cuencas hídricas debido al vertido de efluentes con altos contenidos de metales pesados. La Resolución $N^{\circ}$ 023-2016-OEFA/TFASEM del Ministerio del Ambiente (MINAM) hace referencia a los metales pesados como uno de los grupos de contaminantes ambientales más peligrosos debido a que no son biodegradables y a su potencial de acumulación [1], considerando que la mayoría no tienen funciones metabólicas específicas, de modo que no pueden ser ni degradados ni removidos de manera sencilla, afectando la biodiversidad de ríos y lagos y produciendo en muchos casos la mortalidad de los organismos vivos presentes en dichos ecosistemas [2,3].

Los metales pesados pueden ser transportados en los cuerpos de agua, filtrarse a través del suelo, y en el peor de los casos llegar a las fuentes de agua potable y a los cultivos

Digital Object Identifier: (to be inserted by LACCEI).

ISSN, ISBN: (to be inserted by LACCEI). alimentarios, incorporándose en la cadena trófica y en última instancia alcanzar al ser humano, con severas consecuencias sobre la salud al ser ingeridos en altas concentraciones, causando enfermedades como erupciones cutáneas, hemorragia nasal, problemas respiratorios, sistema inmunológico suprimido, enfermedades hepáticas, asma, anemia, lesiones de piel, diabetes, neurotoxicidad, nefrotoxicidad, enfermedades cardiovasculares, osteoporosis, cáncer, entre otras $[2,3,4]$.

En el Perú, es recurrente que actividades industriales como la minería, curtiduría de pieles, fabricación de pinturas, galvanizado de metales, etc., viertan efluentes con altas concentraciones de metales como el plomo $(\mathrm{Pb})$, cromo $(\mathrm{Cr})$, cobre $(\mathrm{Cu})$, hierro $(\mathrm{Fe})$, arsénico (As), etc. en las cuencas hídricas [5], superando en muchos casos los límites máximos permitidos por el D.S. 004-2017-MINAM [6]. Como alternativa de tratamiento de dichos efluentes se aplican los tradicionales procesos físicos de membrana, tratamientos químicos de intercambio iónico o con carbón activado, precipitación, coagulación, etc. [7], los cuales sin embargo requieren de una inversión tecnológica que en muchos casos los hacen económicamente inviables.

Como alternativa eco-amigable, en el Perú es considerable la producción de residuos alimentarios que no son aprovechados adecuadamente, por ejemplo cáscaras de frutas y hortalizas, residuos de cereales, etc. Diferentes investigaciones han sido encaminadas en el uso de estos residuos como material adsorbente para remover metales de aguas contaminadas [2,8], aprovechando la interacción entre los iones metálicos y los polímeros constituyentes de estos residuos agroindustriales mediante el fenómeno de adsorción química [9]. Entre los polímeros que tienen la capacidad de adsorber iones metálicos se encuentran la pectina, presente en una amplia variedad de cáscaras de frutas, hortalizas y otras biomasas vegetales, así como la lignina. Ambos polímeros contienen grupos hidroxilo $(\mathrm{OH})$, carbonilo $(\mathrm{CO})$, carboxilo $(\mathrm{COOH})$, etc., con capacidad de fijar iones metálicos $[9,10]$.

En tal sentido, el objetivo del presente estudio fue evaluar la capacidad de remoción de plomo $(\mathrm{Pb})$ de aguas contaminadas mediante cáscara de plátano, teniendo en cuenta

16 $^{\text {th }}$ LACCEI International Multi-Conference for Engineering, Education, and Technology: "Innovation in Education and Inclusion", 19-21 July 2018, Lima, United States. 
el tamaño de partícula y el tiempo de contacto entre el adsorbente y el medio contaminado con $\mathrm{Pb}$, considerando la disponibilidad y bajo costo de este residuo alimentario.

\section{MATERIALES Y MÉTODOS}

La metodología propuesta para el desarrollo de las pruebas experimentales está basada en procedimientos recogidos de las referencias bibliográficas citadas en las respectivas secciones, así como en ensayos previos llevados a cabo con el fin de determinar los diferentes parámetros aplicables de acuerdo a la Fig. 1. El detalle de cada etapa se describe en las secciones subsiguientes.

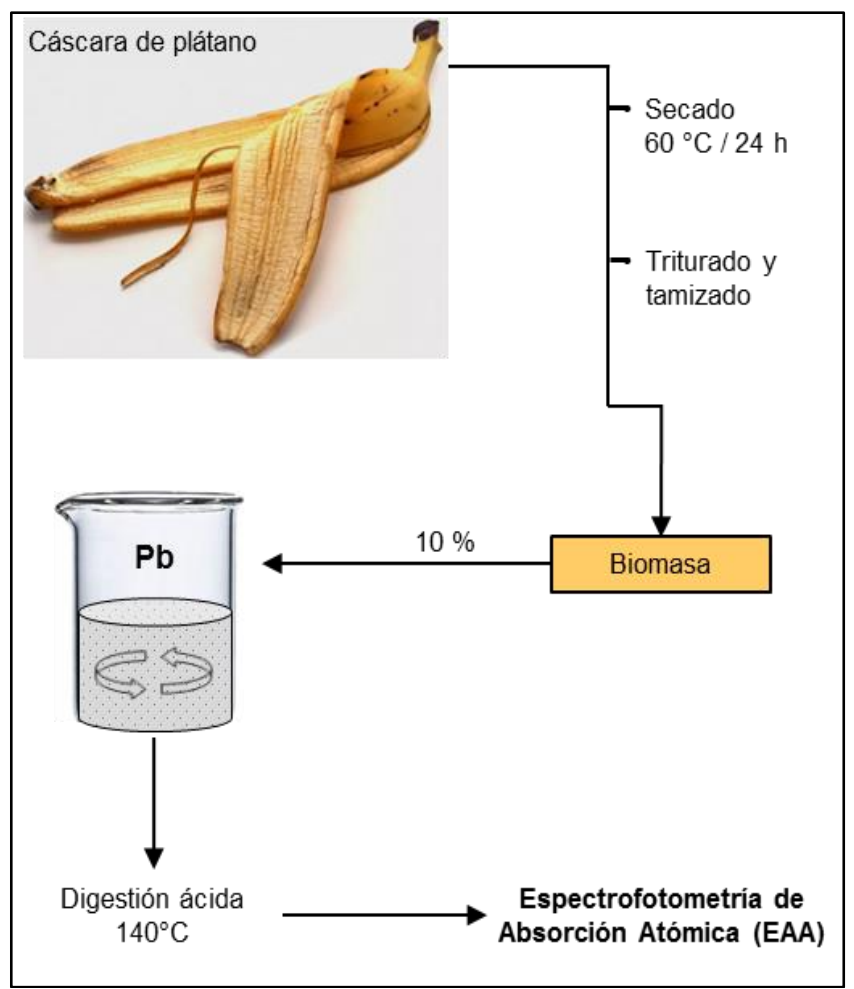

Fig. 2 Procedimiento experimental para la remoción de plomo $(\mathrm{Pb})$ en medios contaminados mediante biomasa de cáscara de plátano.

\section{A. Preparación del adsorbente a base de cáscara de plátano}

Se procedió a lavar la cáscara de plátano (CP) con agua destilada y cortarlo en trozos de $1 \mathrm{~cm}$ de lado, para posteriormente ser secado en estufa de convección forzada a $60^{\circ} \mathrm{C}$ por 24 horas. Posteriormente la cáscara deshidratada fue triturada en un molino eléctrico, y la harina obtenida se tamizó para obtener tamaños de partícula equivalentes a mallas \#20, \#40 y \#60 (aberturas de 841, 400 y $250 \mu \mathrm{m}$, respectivamente).

\section{B. Pruebas experimentales}

Los ensayos se realizaron en el Laboratorio de Análisis Instrumental de la Facultad de Ingeniería de la Universidad Privada del Norte (UPN) sede Trujillo.
Se preparó un medio modelo cuya concentración inicial fue de $50 \mathrm{ppm}$ de $\mathrm{Pb}$ con un $\mathrm{pH}$ inicial de 4.80, a partir de un estándar comercial (Merck Millipore, Alemania) disuelto en agua destilada. Dicho medio fue colocado en diferentes recipientes, considerando los tamaños de partícula y los tiempos de contacto, de acuerdo al diseño experimental mostrado en la tabla 1. A cada recipiente se adicionó la harina de cáscara de plátano (biomasa $\mathrm{CP}$ ) a una proporción del $10 \%$ p/v y se dejó en agitación a 320 rpm.

Se aplicó un diseño factorial para la recolección de datos, siendo la variable dependiente la concentración residual de $\mathrm{Pb}$ (o su equivalente en porcentaje de remoción de $\mathrm{Pb}$ ), mientras que el tamaño de partícula (\# malla) y el tiempo de contacto constituyeron las variables independientes, trabajando por triplicado en cada ensayo (27 pruebas en total).

TABLA 1

\begin{tabular}{|c|c|c|c|}
\hline \multirow{2}{*}{$\begin{array}{l}\text { Tiempo de } \\
\text { contacto } \\
\text { (minutos) }\end{array}$} & \multicolumn{3}{|c|}{ Tamaño de partícula (\# malla) } \\
\hline & 20 & 40 & 60 \\
\hline \multirow[t]{3}{*}{30} & Y111 & Y211 & Y311 \\
\hline & Y112 & Y 212 & Y312 \\
\hline & Y113 & Y213 & Y313 \\
\hline \multirow[t]{3}{*}{60} & Y121 & Y221 & Y321 \\
\hline & $\mathrm{Y} 122$ & Y222 & Y322 \\
\hline & Y123 & Y223 & Y323 \\
\hline \multirow[t]{3}{*}{90} & Y131 & Y231 & Y331 \\
\hline & Y132 & Y232 & Y332 \\
\hline & Y133 & Y233 & Y333 \\
\hline
\end{tabular}

C. Digestión de las muestras y análisis del contenido residual de plomo

Las muestras extraídas tras el tratamiento fueron filtradas y digestadas en base a la metodología propuesta por CamposFlores et al. [11], para lo cual se tomaron $25 \mathrm{~mL}$ de cada muestra y se adicionaron $5 \mathrm{~mL}$ de $\mathrm{HNO}_{3}$ y $1 \mathrm{~mL}$ de HCL. Se sometió a digestión a $120{ }^{\circ} \mathrm{C}$ en un digestor DigiPrepJr (SCP Science, Canadá), evaporando el contenido hasta la mitad, tras lo cual se subió la temperatura hasta $140{ }^{\circ} \mathrm{C}$, evaporando hasta $5 \mathrm{~mL}$. Se dejó enfriar y cada muestra se aforó con una solución acidificada al $1 \%$ con $\mathrm{HNO}_{3}$.

El contenido residual de $\mathrm{Pb}(\mathrm{C}: \mathrm{mg} / \mathrm{L}$ o $\mathrm{ppm}$ ) fue determinado mediante Espectrofotometría de Absorción Atómica con aspiración directa (Espectrofotómetro Agilent Technologies 200 Series AA, Estados Unidos), ajustando la longitud de onda de la lámpara a $217 \mathrm{~nm}$. Dicho contenido residual de $\mathrm{Pb}$ fue utilizado para el cálculo del porcentaje de remoción en cada ensayo.

D. Capacidad de adsorción de la biomasa de cáscara de plátano $(C P)$

Se determinó la cantidad de $\mathrm{Pb}$ (adsorbato) adsorbida por cada gramo de biomasa de cáscara de plátano (CP)

16 $^{\text {th }}$ LACCEI International Multi-Conference for Engineering, Education, and Technology: "Innovation in Education and Inclusion", 19-21 July 2018, Lima, United States. 
(adsorbente), expresada como $q$ mediante la siguiente ecuación [2]:

$$
q=V \frac{(C o-C)}{m}
$$

Donde $\mathrm{V}$ es el volumen (litros) de la solución que se ha tomado para realizar el proceso de adsorción, Co es la concentración inicial de $\mathrm{Pb}(\mathrm{mg} / \mathrm{L} \quad \mathrm{o} \quad \mathrm{ppm}), \quad \mathrm{C}$ es la concentración residual de $\mathrm{Pb}(\mathrm{mg} / \mathrm{L}$ o ppm), y m es la masa del adsorbente (gramos).

\section{E. Análisis estadístico}

Se realizó un Análisis de Varianza (ANOVA) y la prueba LSD de Fisher (5\% de significancia) para identificar diferencias significativas y determinar los parámetros óptimos de tratamiento utilizando el software Statgraphics v.5 (Graphics Software Systems, Rockville, Estados Unidos).

\section{RESULTADOS Y DISCUSIÓN}

La Fig. 2 muestra las concentraciones residuales de $\mathrm{Pb}$ tras los diferentes tratamientos. De acuerdo al análisis estadístico, se obtuvieron diferencias significativas (LSD Fisher, $\mathrm{p}<0.05)$ en la remoción de $\mathrm{Pb}$ al utilizar partículas equivalentes a la malla \#60 $(250 \mu \mathrm{m})$. Si bien para todos los tamaños de partícula se hicieron tratamientos de hasta 90 minutos, de acuerdo a los resultados obtenidos bastaría un tiempo de contacto de 30 minutos para remover la mayor cantidad de $\mathrm{Pb}$ del medio contaminado, tal como se muestra en la tabla 2, al alcanzar una remoción en torno al $99 \%$ a este tiempo de tratamiento.

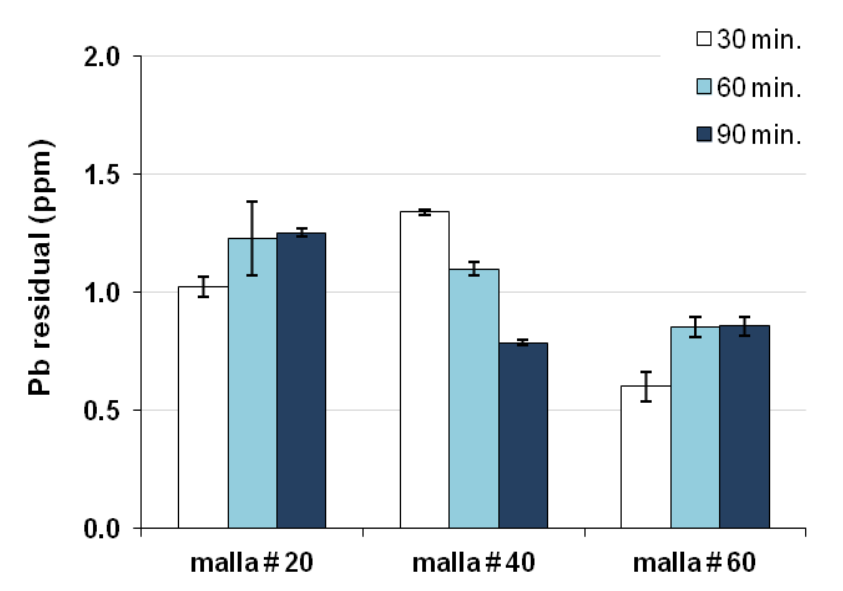

Fig. 2 Concentraciones residuales de plomo $(\mathrm{Pb})$ tras los tratamientos (concentración inicial: $50 \mathrm{ppm}$ ).

Los resultados obtenidos indican una mayor adsorción de $\mathrm{Pb}$ con el menor tamaño partícula (malla \#60: $250 \mu \mathrm{m}$ ). Se conoce que un mayor tamaño de partícula ofrece mayor número de puntos de contacto adsorbente-adsorbato, es decir una mayor capacidad de adsorción. No obstante, otro parámetro involucrado es la intensidad de adsorción [8], el cual depende de la intensidad mediante la cual los grupos funcionales presentes en el adsorbente pueden fijar al ion metálico, determinado por las propiedades químicas de la cáscara de plátano, con alto contenido de grupos carboxilo ($\mathrm{COOH})$, que forman parte de la estructura química de la pectina [12,13]. De modo que la mayor remoción de $\mathrm{Pb}$ obtenida con la malla \#60 indicaría un mayor efecto de las interacciones entre la pectina y los iones de $\mathrm{Pb}$, más que del número de puntos de contacto entre ambos.

TABLA 2

PORCENTAJES DE REMOCIÓN DE PLOMO (Pb). CONCENTRACIÓN INICIAL: 50 PPM.

\begin{tabular}{|c|c|c|c|}
\hline \multirow{3}{*}{$\begin{array}{l}\text { Tiempo de } \\
\text { contacto } \\
\text { (min.) }\end{array}$} & \multicolumn{3}{|c|}{ Remoción de Pb (\%) } \\
\hline & \multicolumn{3}{|c|}{ \# de malla } \\
\hline & 20 & 40 & 60 \\
\hline 30 & $97.95 \pm 0.08^{\mathrm{a}, \mathrm{B}}$ & $97.32 \pm 0.02^{\mathrm{c}, \mathrm{C}}$ & $98.80 \pm 0.12^{\mathrm{a}, \mathrm{A}}$ \\
\hline 60 & $97.54 \pm 0.31^{\mathrm{ab}, \mathrm{B}}$ & $97.80 \pm 0.05^{\mathrm{b}, \mathrm{B}}$ & $98.29 \pm 0.08^{\mathrm{b}, \mathrm{A}}$ \\
\hline 90 & $97.49 \pm 0.03^{\mathrm{b}, \mathrm{c}}$ & $98.43 \pm 0.02^{\mathrm{a}_{\mathrm{a}} \mathrm{A}}$ & $98.29 \pm 0.08^{\mathrm{b}, \mathrm{B}}$ \\
\hline \multicolumn{4}{|c|}{$\begin{array}{l}\text { Diferentes letras minúsculas en la misma columna indican diferencia } \\
\text { significativa a diferentes tiempos de contacto. Diferentes letras } \\
\text { mayúsculas en la misma fila indican diferencia significativa a } \\
\text { diferentes \#malla. Test LSD de Fisher }(\mathrm{p}<0.05) \text {. }\end{array}$} \\
\hline
\end{tabular}

Los mejores resultados obtenidos con el menor tiempo de contacto, es decir a los 30 minutos, indicaría que a ese tiempo se alcanzó la saturación de los grupos - $\mathrm{COOH}$ que actúan como quelantes de los iones de $\mathrm{Pb}$, con lo cual el sistema adsorbente-adsorbato alcanzaría el equilibrio [14], es decir, la biomasa $\mathrm{CP}$ ya no es capaz de adsorber más $\mathrm{Pb}$ a partir de ese momento [11].

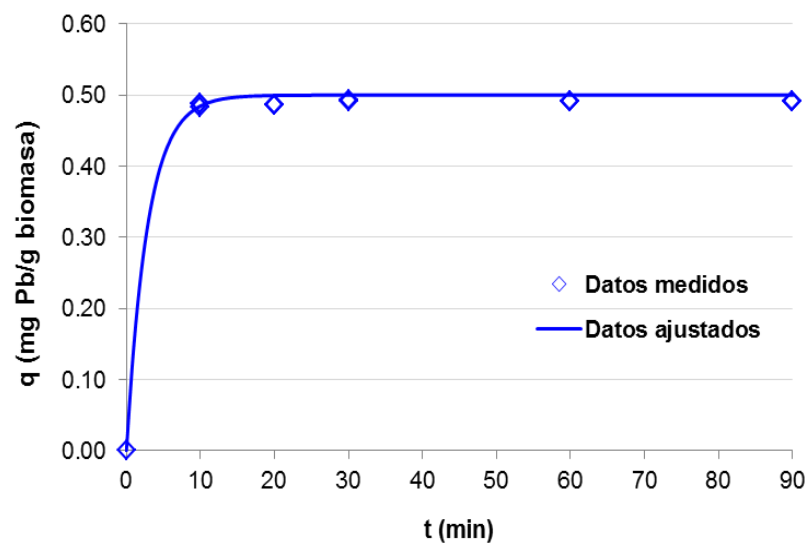

Fig. 3 Capacidad de adsorción (q: mg Pb/g biomasa CP) a un tamaño de partícula de $250 \mu \mathrm{m}$ (malla \#60). Datos ajustados con el software SIMFIT (The University of Manchester, United Kingdom).

Obtenido el tamaño óptimo de partícula (malla \#60), se procedió a determinar la capacidad de adsorción $(q: \mathrm{mg} / \mathrm{g})$, es decir la cantidad de $\mathrm{Pb}$ retenida por cada gramo de biomasa CP [2], adicionando dos tiempos de contacto (10 y 20 minutos en agitación), cuyos resultados se muestran en la Fig. 3. Obteniendo que la máxima adsorción de $\mathrm{Pb}$ se logra a partir de 
los 30 minutos, con un valor aproximado de $q$ de $0.49 \mathrm{mg}$ $\mathrm{Pb}$ /gramo de biomasa $\mathrm{CP}$, tiempo en el cual se alcanzaría la saturación de la biomasa de cáscara de plátano.

Finalmente, es conocida la problemática asociada al inadecuado control de vertidos de metales en las cuencas hídricas. Por ejemplo, la elevada concentración de metales pesados en el río Moche en La Libertad [5], se ha relacionado con su alta presencia en cultivos ubicados en dicha cuenca, lo cual representa un peligro para la salud debido a la ingesta progresiva de metales como el $\mathrm{Pb}$ hasta niveles tóxicos en el organismo.

\section{CONCLUSIONES}

Se determinó la eficacia de la biomasa de cáscara de plátano $(\mathrm{CP})$ para la remoción de $\mathrm{Pb}$ mediante el mecanismo de adsorción, sugiriendo nuestros resultados que se puede reducir en más del $95 \%$ el contenido de este metal en los medios líquidos ensayados. La mayor remoción de $\mathrm{Pb}$ se obtuvo con la malla \#60 $(250 \mu \mathrm{m})$ a un tiempo de contacto de 30 minutos, logrando remover más del $98 \%$ de $\mathrm{Pb}$ del medio contaminado bajo las condiciones del estudio. En general la adsorción mediante la cáscara de plátano constituye una potencial alternativa a los métodos convencionales de remoción de metales en aguas contaminadas, por lo que se puede utilizar como una tecnología efectiva, de fácil manejo y amigable con el ambiente. Finalmente, es necesario el desarrollo de futuros trabajos respecto a la aplicabilidad de la biomasa de cáscara de plátano como adsorbente a diferentes concentraciones iniciales de $\mathrm{Pb}$, a fin de determinar parámetros óptimos de remoción como la capacidad máxima de adsorción $\left(q_{\max }\right)$ mediante el modelo de Langmuir, así como la capacidad $(k)$ e intensidad de adsorción $(n)$ mediante el modelo de Freundlich; además de evaluar la efectividad de esta biomasa como adsorbente para la remoción de $\mathrm{Pb}$ en efluentes industriales, como los producidos por la minería, con el fin de validar los resultados obtenidos a nivel de laboratorio, y desarrollar un procedimiento de remoción con perspectivas de aplicabilidad a nivel industrial.

\section{AGRADECIMIENTOS}

Este trabajo forma parte del Proyecto 20171002: "Remoción de metales pesados en aguas contaminadas mediante residuos agroindustriales", de la Universidad Privada del Norte (UPN).

\section{REFERENCIAS}

[1] OEFA. 2016. RESOLUCION No 023-2016-OEFA/TFA-SEM. Tribunal de Fiscalización Ambiental, Sala Especializada en Minería. Ministerio del Ambiente. Pág. 18-19. Disponible en: https://www.oefa.gob.pe/?wpfb_dl=17626.

[2] Ali, A., Saeed K., Mabood, F. 2016. Removal of chromium (VI) from aqueous médium using chemically modified banana peels as efficient low-cost adsorbent. Alexandria Engineering Journal, 55: 2933-2942.

[3] Sud, D., Mahajan, G., Kaur, M. 2008. Agricultural waste material as potential adsorbent for sequestering heavy metal ions from aqueous solutions: a review. Bioresource Technology, 99: 6017-6027.

[4] Squadrone, S., Burioli, E., Monaco, G., Koya, M.K., Prearo, M., Gennero, S., Dominici, A., Abete, A.C. 2016. Human exposure to metals due to consumption of fish from an artificial lake basin close to an active mining area in Katanga (D.R. Congo). Science of the Total Environment, 568: 679-684.

[5] Huaranga, F., Méndez, E., Quilcat, V., Huaranga, F. 2012.Contaminación por metales pesados en la cuenca del río Moche, 1980 - 2010, La Libertad - Perú. Scientia Agropecuaria, 3(3): 235-247.

[6] MINAM. 2017. Decreto Supremo $\mathrm{N}^{\circ}$ 004-2017-MINAM. Aprueban Estándares de Calidad Ambiental (ECA) para Agua y establecen Disposiciones Complementarias. Ministerio del Ambiente (MINAM). Disponible en: http://www.minam.gob.pe/disposiciones/decreto-supremon-004-2017-minam/.

[7] Gupta, V.K., Kumar, R., Nayak, A., Saleh, T.A., Barakat, M.A. 2013. Adsorptive removal of dyes from aqueous solution onto carbon nanotubes: a review. Advances in Colloid and Interface Science, 193-194: 24-34.

[8] Tejada-Tovar, C., Villabona-Ortiz, A., Garcés-Jaraba, L. 2015. Adsorption of heavy metals in waste water using biological materials. TecnoLógicas, 18(34): 109-123.

[9] Iliná, A., Martínez, J., Segura, E., Villarreal, J., Gregorio, K. 2009. Biosorción de arsénico en materiales derivados de maracuyá. Revista Internacional de Contaminación Ambiental, 25(4): 201-216.

[10]Pehlivan, E., Altun, T., Parlayici, S. 2012. Modified barley straw as a potential biosorbent for removal of copper ions from aqueous solution. Food Chemistry, 135(4): 2229-2234.

[11] Campos-Flores, G., Castillo-Herrera, A., Gurreonero-Fernández, J., Obeso-Obando, A., Díaz-Silva, V., Vejarano, R. 2017. Adsorbent material based on passion-fruit wastes to remove lead $(\mathrm{Pb})$, chromium $(\mathrm{Cr})$ and copper $(\mathrm{Cu})$ from metal-contaminated waters. AIP Conference Proceedings, 1952: 020079.

[12] Chao, H.P., Chang, C.C., Nieva, A. 2014. Biosorption of heavy metals on Citrus maxima peel, passion fruit shell, and sugarcane bagasse in a fixedbed column. Journal of Industrial and Engineering Chemistry, 20 (5): 3408-3414.

[13]Hu, M., Zhang, S., Pan, B., Zhang, W., Lv, L., Zhang, Q. 2012. Heavy metal removal from water/wastewater by nanosized metal oxides: a review. Journal of Hazardous Materials, 211-212: 317-331.

[14]Jacques, R., Lima, E., Dias, S., Mazzocato, A., Pavan, F. 2007. Yellow passion-fruit shell as biosorbent to remove $\mathrm{Cr}$ (III) and $\mathrm{Pb}$ (II) from aqueous solution. Separation and Purification Technology, 57: 193-198.

$16^{\text {th }}$ LACCEI International Multi-Conference for Engineering, Education, and Technology: "Innovation in Education and Inclusion", 19-21 July 2018, Lima, United States. 